\title{
ESTIMATION OF CRITICAL GAP AT MID-BLOCK MEDIAN OPENINGS
}

\author{
Aparna Vimal Kumar', Salini Sasikumar ${ }^{2}$ \\ ${ }^{1,2}$ Department of Civil Engineering, College of Engineering Trivandrum, Thiruvananthapuram, Kerala, India
}

Received 2 July 2020; accepted 10 August 2020

\begin{abstract}
U-turning movements impart a significant effect on traffic characteritics at the medians.This paper attempts to study critical gap of U-turns at unsignalized median openings. Mixed traffic conditions in India makes critical gap estimation more tedious task. Critical gap at median openings was estimated using traditional method, modified Raff method and merging behaviour approach. The merging behaviour approach was found to be more suitable under heterogeneous conditions. The estimation of critical gap by merging behaviour approach is done based on the merging time of U-turning vehicles. A model was developed for predicting critical gap considering all vehicle types. From the analysis the critical gap was found to depend on accepted gap and merging time.
\end{abstract}

Keywords: U-turn, critical gap, merging behaviour method.

\section{Introduction}

Road transport is the most accessible mode of transport. Rapid urbanisation has resulted into severe congestion in Indian roads. The traffic in India is characterized by mixed traffic condition and weak lane discipline. The urban roads are constructed as four lanes or six lane to meet the heavy traffic demand. These multilane roads are constructed with medians to segregate opposing traffic movements. As part of traffic management to improve intersection operation minor movements like U-turning movements are prohibited at selected intersections. Median openings are provided at adequate intervals to reverse the direction of travel. The increased installation of restrictive medians and directional median openings has increased the number of U-turns on multilane highways. U-turning movement at median openings are highly complex and risky. The U-turning vehicle has to make a $180^{\circ}$ turning movement to merge with opposing traffic. The high speed and heavy traffic volume of opposite flow also makes the U-turning difficult. As the number of U-turning vehicles increase queuing condition occurs and then a long queue will be formed and the queue spill back to block through traffic stream. This leads to traffic problems like reduced capacity and level of safety.

For a safe U-turn, the U-turning vehicle has to accept a suitable gap between the arrivals of successive vehicles in the through traffic of opposing lane. The gap acceptance behaviour of vehicle is mainly analysed by using the parameter, critical gap. The critical gap is defined as the minimum gap that is acceptable to a driver, anticipating merging

${ }^{2}$ Corresponding author: salinisasikumar@cet.ac.in 
with the approaching through traffic stream. HCM (2010) defines critical headway as "minimum time interval between the front bumpers of two successive vehicles in the major traffic stream that will allow the entry of one minor street vehicle".

U-turning vehicles have to wait for acceptable gap and merge with approaching traffic when sufficient gap is obtained. But the rule of priority is often violated and forced gap acceptance occurs in mixed traffic conditions. The U-turning vehicles accepts shorter gaps forcing opposing through traffic to slow down. The forced gap acceptance under mixed traffic condition is due to impatient and discourteous behaviour of drivers. Forced gap acceptance will change the behaviour of opposing through traffic also. These adverse conditions make critical gap estimation under heterogeneous conditions more difficult. The present study attempts to estimate and model critical gap of U-turns in mid-block median openings under heterogeneous traffic flow conditions.

\section{Literature Review}

Liu et al. (2007) studied headway acceptance characteristics of U-turning vehicles at unsignalized intersections. Maximum likelihood method was used for critical headway estimation. The authors found that median width affects the decision of U-turning drivers, whether to accept or reject a particular headway. Results of the study indicated that critical headway is smaller at wide medians compared with narrow medians. Ashalatha and Chandra (2011) estimated critical gap at unsignalised intersections by some of the existing methods like Lag, Harder, Logit, Probit, modified Raff and Hewitt methods. The results showed that critical gap values are very low (1.60 s) and significant variation (12\%-38\%) was observed among the values estimated by different techniques. They developed a new concept for critical gap estimation by assessing the clearing behaviour of vehicles. They identified an area Influence Area for Gap Acceptance (INAFOGA) at unsignalized intersection. Jenjiwattanakul and Sano (2011) investigated the effect of waiting time in gap acceptance behaviour of U-turning vehicles. The authors found that longer waiting times ( $>30$ seconds) would frustrate the drivers and they accept shorter gaps which once they rejected. Obaidat and Elayan (2013) developed two models, one model to estimate gap needed for U-turning drivers and second model predicts whether a U-turning driver accepts a particular gap. First model estimates length of gap required by a driver based on their age, gender and waiting time. A U-turning choice model was developed, which gives utility of turning based on gap length, age of the driver and waiting time. The utility function was used to determine probability of U-turning.

Datta (2014) compared Harder's and INAFOGA method. A paired sample t-test was done, which revealed that INAFOGA method yield critical gap values $28-41 \%$ more than those obtained by Harder's method. Harder's method was found to be inefficient in estimating critical gap values under heterogeneous traffic conditions. Datta and Bhuyan (2014) estimated critical gap of U-turning vehicles by INAFOGA and Macroscopic Probability Equilibrium. The critical gap values obtained by INAFOGA were found to be $18-31 \%$ more than those obtained by probability equilibrium method. Khan et al. (2015) studied critical gap values of U-turning vehicles at the upstream and downstream of median opening. The difference in critical gap values 
for upstream and downstream U-turning vehicles was less in the case of Raff's method, Greenshield's method, acceptance curve method and Harder's method. But the models like maximum likelihood estimation and Ningwu's method yielded large variation in critical gap values for upstream and downstream traffic. The results showed that critical gap values obtained by Greenshield and Ashworth's method are consistent.

Paneela and Bhuyan (2016) estimated critical gap by Modified Raff method and Harder's method. This showed the limitation of existing methods in estimation of critical gap under heterogeneous traffic conditions. The authors compared INAFOGA method with probability equilibrium method. Difference in critical gap value indicated that the probability equilibrium method is not suitable under mixed traffic conditions. Dash et al. (2019) estimated critical gap at uncontrolled median openings by maximum likelihood method, Modified Raff method, and macroscopic probability equilibrium method. Authors proposed merging behaviour method, it was found to be suitable in assessing critical gap under mixed traffic conditions. Sharma et al. (2017) estimated critical gap at median openings in six lane roads based on traditional method, Modified Raff method and INAFOGA method. Critical gap values obtained by INAFOGA method was about $5.93 \%$ higher than traditional method and about $16.38 \%$ higher than Modified Raff method. The authors proposed a model considering all motorised mode as common mode where the critical gap value depends on merging time accepted gaps.

\section{Methodology}

Critical gap is an important parameter to study the gap acceptance behaviour of U-turning movement. It is very difficult to measure critical gap directly from field. However accepted and rejected gaps can be directly measured from field. In the present study three different methodologies namely traditional method, modified Raff method and merging behaviour approach were used for critical gap estimation.

\subsection{Traditional Method}

In traditional method cumulative number of accepted and rejected gaps is plotted. The point of intersection of these two gaps is reported as critical gap. Lag data is not considered for critical gap estimation.

\subsection{Modified Raff Method}

Modified Raff method uses both lag and gap for critical gap estimation. The modified Raff method involves the observed distribution function of accepted gaps $F_{a}(t)$ and rejected gaps $F_{r}(t)$. When the sum cumulative probabilities of accepted gaps and rejected gaps are equal to 1.0 , then a gap of length $t$ is equal to critical gap $t_{c}$. This means that number of rejected gaps larger than critical gap is equal to the number of accepted gaps smaller than critical gap. The mathematical expression for the critical gap is presented below:

$F_{a}(t)=1-F_{r}(t)$

The cumulative frequency distribution curves of accepted and rejected gaps are 
plotted and the point of intersection of the two curves gives the critical gap.

\subsection{Merging Behaviour Approach}

Dash et al. (2019) proposed merging behaviour approach for critical gap estimation at median openings. An area $A B C D$, was identified at the median opening as INAFOGA for the U-turning vehicle. Figure 1 shows schematic diagram of conflict zone at median opening. The reference line $A B$ was taken as the stop line at median opening where the U-turning vehicle stops before merging with the opposing through traffic stream. The upstream boundary area $\mathrm{BC}$ is taken as half of the median width. The lower boundary CD depends on critical position of U-tuning vehicle. The identification of boundaries $\mathrm{BC}$ and $\mathrm{CD}$ have less significance in the estimation of merging time. The boundary DA is considered as merging line it varies with vehicle category. Merging line was not considered as a fixed line in this study, since the merging of different vehicles within the same vehicle category varies.

Lag was measured as the time interval between arrival of a U-turning vehicle at $\mathrm{AB}$ and the arrival of next through traffic vehicle at reference line $\mathrm{BC}$. $\mathrm{BC}$ was taken as reference line for estimation of gap. Accepted gap and lag were noted. The merging time was taken as the time when the rear bumper passed over line $A B$ to the rear bumper crossed merging line AD.

The cumulative frequency distribution curves of merging time and the corresponding lag and gap acceptance cumulative frequency curves were plotted for each category of vehicle. The point of intersection of these two curves was reported as the critical gap.

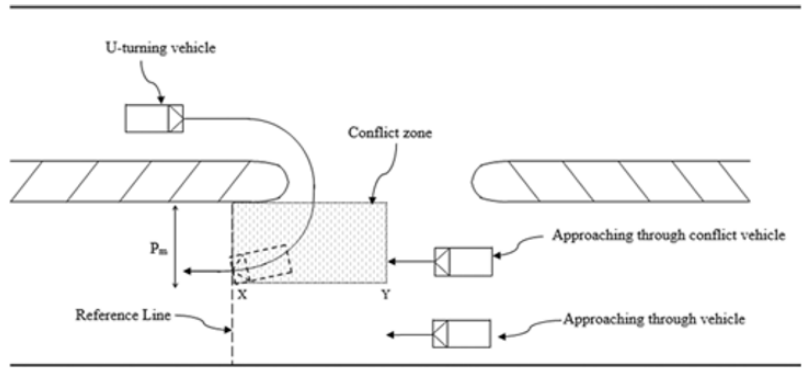

Fig. 1.

Schematic Diagram of Conflict Zone at Median Opening

Source: (Mohapatra et al., 2015)

\section{Study Area}

The traffic data were collected from mid-block median openings in Thiruvananthapuram and Ernakulum city in Kerala, India. In Thiruvananthapuram city, there are number of locations with significant U-turning traffic. Most of the median openings in Thiruvanantapuram city have U-turn as well as right turns due to the presence of side roads. This right turning traffic may affect the U-turning behaviour. In Thiruvananthapuram city median openings without side roads are less. In Ernakulam city there are number 
of median openings exclusively for U-turn along Kochi metro line. Hence traffic data was also collected from sections in Ernakulam city, where median openings serve U-turn provision only. Fig. 2 shows a typical data collection site in Thiruvananthapuram city.

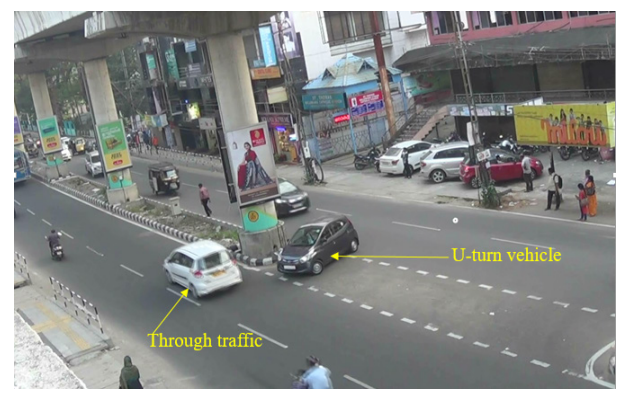

Fig. 2.

Snapshot of Study Section at Maharajas, Ernakulam

Source: (Photo by Authors, 2018)

\section{Data Collection and Extraction}

Videography technique was used to collect data from all the sites. Road sections with different widths were selected for the study. Video datas were collected from straight road segments. Sites free from pedestrian activities, gradient, curves, parking and far from intersection where preferred. Total six median openings in Trivandrum and Ernakulam city in Kerala were selected for the study. Video data were collected from locations with and without side roads for the study.

Gap variables such as lag, accepted gap, rejected gap and merging time were extracted using AVS video editor. The following details were extracted from the video.
1. $\mathrm{T}_{1}=$ time instant front bumper touches the stop line at the median opening (Fig 4 a);

2. $\mathrm{T}_{2}=$ time instant front bumper of first through traffic vehicle after arrival of U-turn vehicle touches the reference line for gap estimation at the upstream of median opening (Fig $4 \mathrm{~b}$ );

3. $\mathrm{T}_{3}, \mathrm{~T}_{4}, \ldots \mathrm{T}_{\mathrm{n}}=$ corresponding time instants for arrival of through traffic on the reference line at the upstream of median opening;

4. $\mathrm{T}_{\mathrm{w}}=$ time instant at which back bumper of the U-turn vehicle touches the stop line at the median opening (Fig $4 \mathrm{c}$ );

5. $\mathrm{T}_{\mathrm{m}}=$ time instant at which back bumper of U-turn vehicle merges with through traffic at the downstream of median opening (Fig $4 \mathrm{~d}$ ). 


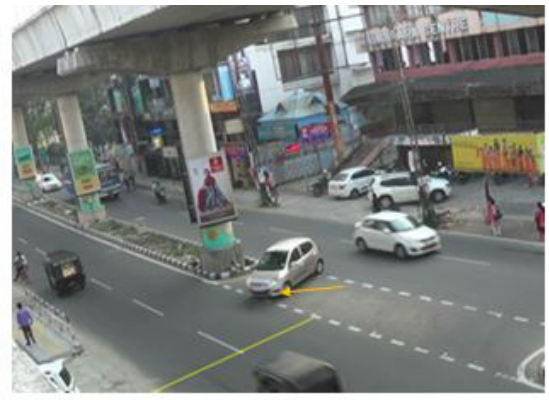

(a)

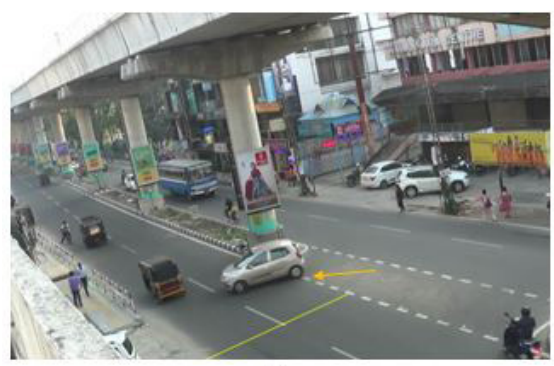

(c)

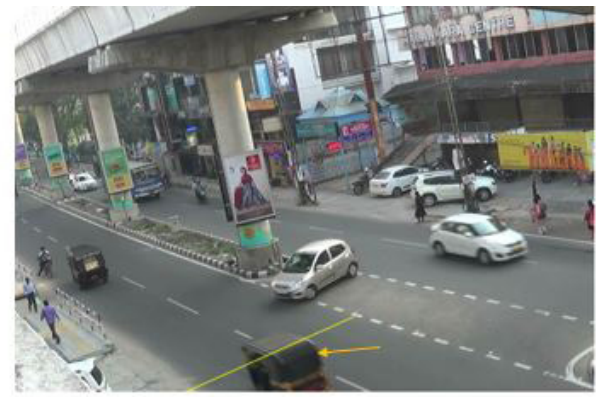

(b)

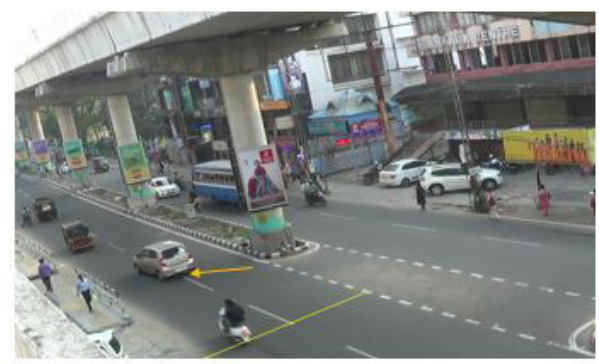

(d)

Fig. 3.

Data Extraction using AVS Video Editor

Source: (Photo by Authors, 2018)

From the above observations lag, accepted gap, rejected gap and merging times were computed as below (Paneela and Bhuyan, 2017):

- $\quad$ Lag $=$ time interval between arrival of U-turn vehicle on median opening and arrival of first through traffic vehicle $=\mathrm{T}_{2}-\mathrm{T}_{1}$;

- $\quad$ Gap (accepted and rejected $)=$ difference between arrivals of successive through traffic vehicles at the reference line for gap estimation at the upstream of the median opening $=T_{n+1}-T_{n}$;

- Merging time of U-turning vehicle = Tm - Tw;

The lag may be rejected or accepted by U-turn vehicle. If the lag is rejected, then gap is considered. Again, the gap may also be rejected or accepted by the U-turn vehicle. It was continued until a gap was ultimately accepted by the U-turn vehicle. Therefore, the U-turn vehicle will probably be searching for a gap in the through traffic to take a suitable turn.

\section{Data Analysis}

Field data was collected from three locations in Thiruvananthapuram city and three sections in Ernakulum city. Table 1 shows the geometric details of the study locations. Table 2 shows the through traffic and U-turning traffic volume at selected study locations. 


\section{Table 1}

Geometric Details of Study Sections

\begin{tabular}{|c|c|c|c|c|}
\hline $\begin{array}{c}\text { Section } \\
\text { ID }\end{array}$ & Road Section & $\begin{array}{c}\text { Width of Road } \\
\text { in one Direction } \\
(\mathbf{m})\end{array}$ & $\begin{array}{c}\text { Width of Median } \\
(\mathbf{m})\end{array}$ & $\begin{array}{c}\text { Width of Median } \\
\text { Opening (m) }\end{array}$ \\
\hline ID 1 & M. G. Road - Maharajas & 6.8 & 2.6 & 14.6 \\
\hline ID 2 & Palarivattom - Changampuzha & 7.8 & 2.4 & 17.0 \\
\hline ID 3 & Pulinchodu - Companypady & 10.5 & 3.0 & 23.9 \\
\hline ID 4 & Killipalm - Karamana & 7.5 & 0.7 & 12.5 \\
\hline ID 5 & Pattoor - General hospital & 7.5 & 0.3 & 12.0 \\
\hline ID 6 & Paruthippara - Kesavadasapuram & 6.0 & 0.3 & 13.6 \\
\hline
\end{tabular}

Table 2

Traffic Volume at Selected Locations

\begin{tabular}{|c|c|c|}
\hline Section ID & $\begin{array}{c}\text { Through Traffic } \\
(\mathbf{P C U} / \mathbf{h})\end{array}$ & $\begin{array}{c}\text { U-turning Traffic } \\
(\mathbf{P C U} / \mathbf{h})\end{array}$ \\
\hline ID 1 & 1737 & 399 \\
\hline ID 2 & 2126 & 417 \\
\hline ID 3 & 3041 & 117 \\
\hline ID 4 & 4923 & 160 \\
\hline ID 5 & 1890 & 339 \\
\hline ID 6 & 2376 & 129 \\
\hline
\end{tabular}

The U-turning traffic composition observed at selected study sections are shown in Table 3. The proportion of two wheelers is found to be more at all the selected sites. U-turns of heavy vehicles were not observed at the study sites and so it was not considered in the present study.

Table 3

U-turning Traffic Composition at Selected Locations

\begin{tabular}{|c|c|c|c|c|c|}
\hline \multirow{2}{*}{ Section ID } & \multicolumn{5}{|c|}{ Composition of U-turning Traffic (\%) } \\
\cline { 2 - 6 } & $\mathbf{2 - W}$ & $\mathbf{3 - W}$ & Car & Big Car & LCV \\
\hline ID 1 & 53.33 & 31.67 & 10.33 & 1.67 & 3.33 \\
\hline ID 2 & 60.53 & 10.90 & 20.82 & 3.87 & 5.08 \\
\hline ID 3 & 40.38 & 9.62 & 30.77 & 7.69 & 1.92 \\
\hline ID 4 & 68.85 & 16.39 & 13.93 & 0 & 0 \\
\hline ID 5 & 41.40 & 35.35 & 20.20 & 0 & 2.02 \\
\hline ID 6 & 44.00 & 32.00 & 24.00 & 0 & 0 \\
\hline
\end{tabular}

\section{Estimation of Critical Gap}

Critical gap at selected median openings were estimated by Traditional method, Modified Raff method and Merging Behaviour approach.

\subsection{Traditional Method}

In Traditional method cumulative frequency distribution curves of accepted and rejected gaps are plotted as shown in Fig. 4 and the point of intersection is taken as critical gap. 
Similarly critical gap values of different wheeler, car, big car and LCV) at different vehicle categories (two wheeler, three sections were estimated.

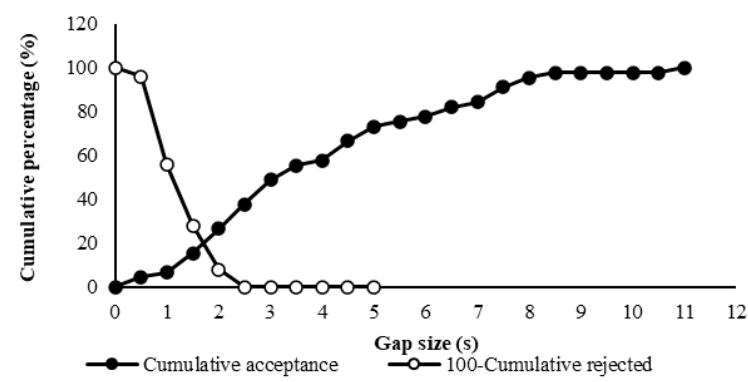

Fig. 4.

Critical Gap Estimation by Traditional Method for $2 W$ at Section 1

\subsection{Modified Raff Method}

In Modified Raff method lag acceptance data was also considered for critical gap estimation. The cumulative distribution curves of accepted gap/lag and rejected gaps for different vehicle categories and different sections are plotted as shown in Fig 5. The intersection point of the two curves is reported as critical gap.

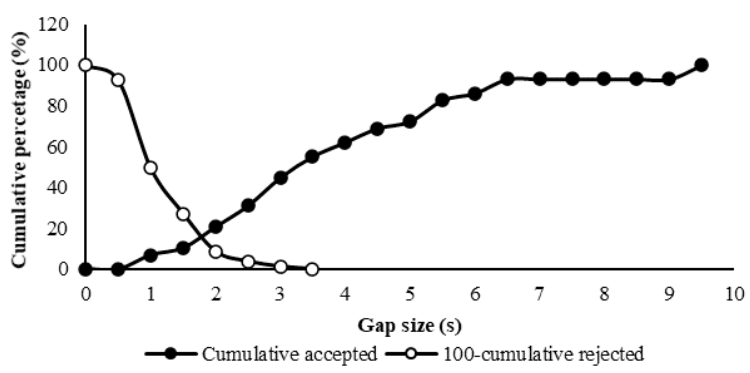

Fig. 5.

Critical Gap Estimation by Modified Raff Method for $2 W$ at Section 2

\subsection{Merging Behaviour Approach}

In Merging Behaviour Approach discrimination between lag and gap were not considered. The cumulative distribution curves of merging time and lag/gap acceptance data of different sites were plotted as shown in Fig. 6. The point of intersection of these two curves gives critical gap. 


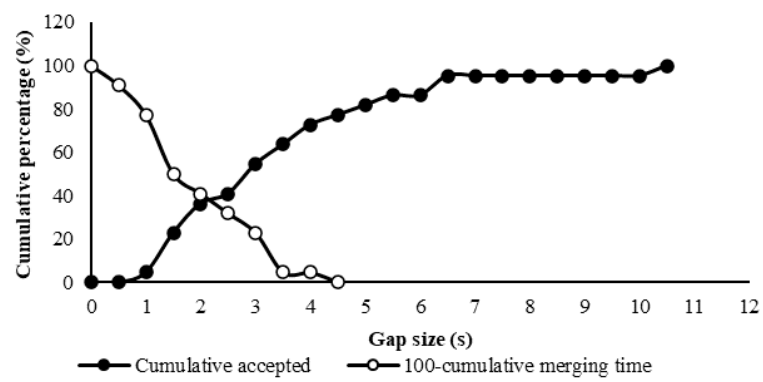

Fig. 6.

Critical Gap Estimation by Merging Behaviour Approach for $2 W$ at Section 3

The critical gap value for different vehicle categories estimated by merging behaviour approach is shown in Fig 7.The critical gap value is found minimum for two wheelers. The variation is attributed by static and dynamic features of the vehicles. The lower critical gap value of two wheelers is due to their smaller dimensions and peculiar driver behaviour.

The critical gap values estimated by traditional, modified Raff and merging behaviour approach are shown in Table 4. Critical gap values of big car and LCV for all the sections were not obtained due to their lower U-turning rate. Critical gap value obtained by traditional method and modified Raff method does not show significant difference. But the critical gap value estimated by merging behaviour approach is found to be more than that obtained by traditional and Modified Raff method.

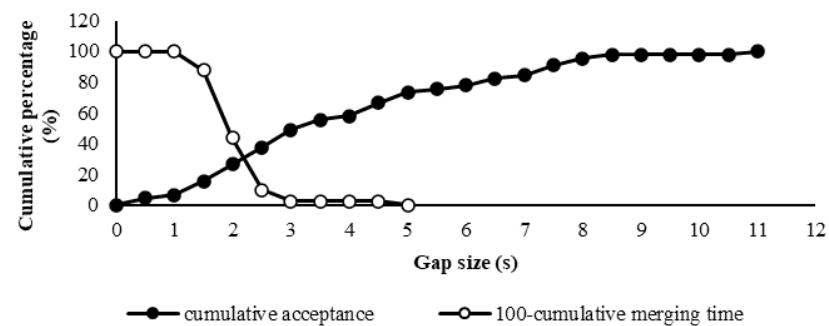

(a)

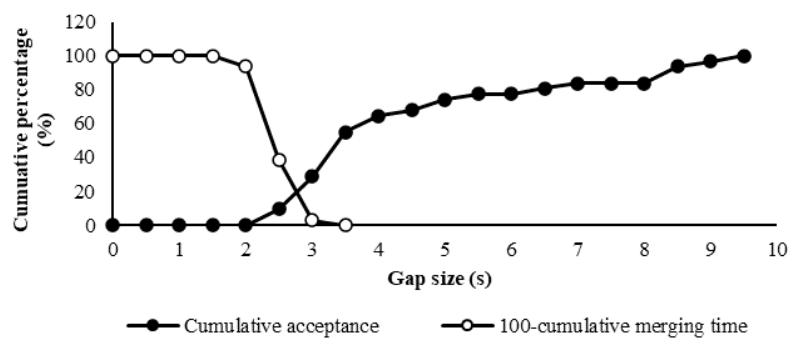

(b) 


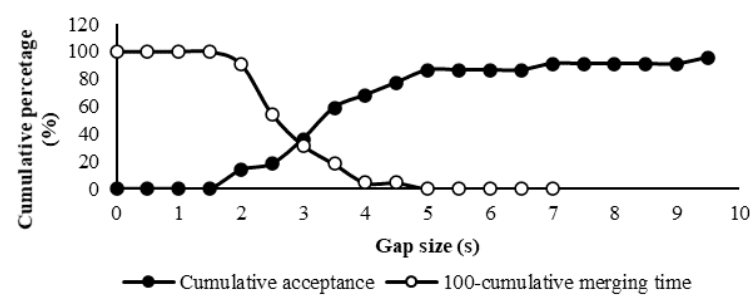

(c)

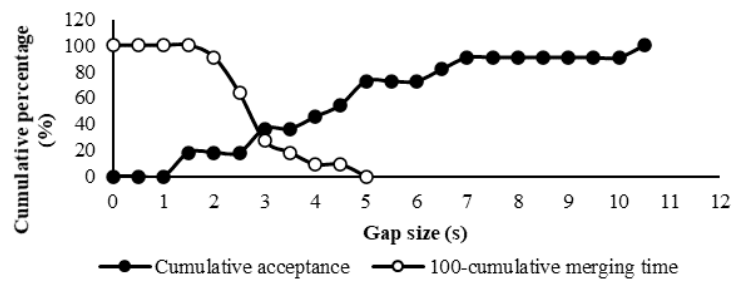

(d)

Fig. 7.

Critical Gap Values Estimated for (a) $2 W($ b) $3 W$ (c) Car (d) LCV at Section 1

Table 4

Critical Gap Values for Different U-turn Vehicle Categories

\begin{tabular}{|c|c|c|c|c|}
\hline Section & Vehicle Type & Traditional & Modified Raff & Merging Behaviour \\
\hline \multirow{5}{*}{ ID 1} & $2 w$ & 1.70 & 1.70 & 2.20 \\
\hline & $3 w$ & 2.25 & 2.25 & 2.75 \\
\hline & Car & 2.50 & 2.50 & 2.95 \\
\hline & Bcar & - & - & - \\
\hline & $\mathrm{LCV}$ & - & 2.80 & 2.90 \\
\hline \multirow{5}{*}{ ID2 } & $2 w$ & 2.00 & 1.75 & 2.35 \\
\hline & $3 w$ & 2.10 & 1.95 & 2.85 \\
\hline & Car & 2.85 & 2.10 & 3.35 \\
\hline & Bcar & 2.70 & 2.00 & 2.90 \\
\hline & $\mathrm{LCV}$ & - & 2.00 & 2.60 \\
\hline \multirow{5}{*}{ ID 3} & $2 w$ & 1.70 & 1.50 & 2.15 \\
\hline & $3 w$ & 2.25 & 2.20 & 3.75 \\
\hline & Car & 2.20 & 2.30 & 3.80 \\
\hline & Bcar & - & - & 3.35 \\
\hline & LCV & - & - & 5.00 \\
\hline \multirow{3}{*}{ ID 4} & $2 w$ & 1.35 & 1.35 & 2.10 \\
\hline & $3 w$ & 1.65 & 1.60 & 2.75 \\
\hline & Car & 2.30 & 2.40 & 3.00 \\
\hline \multirow{3}{*}{ ID 5} & $2 w$ & 2.00 & 2.10 & 2.75 \\
\hline & $3 w$ & 2.50 & 2.40 & 3.50 \\
\hline & Car & 2.85 & 2.90 & 4.15 \\
\hline \multirow{3}{*}{ ID 6} & $2 w$ & 1.50 & 1.50 & 2.25 \\
\hline & $3 w$ & 2.55 & 2.50 & 2.90 \\
\hline & Car & 2.30 & 3.00 & 3.50 \\
\hline
\end{tabular}




\section{Modelling of Critical Gap}

Critical gap was modelled considering five independent variables such as lag, accepted gap, rejected gap, merging time and conflicting traffic volume. A base model was formed considering all five variables.

For accepted gap, lag and merging time $t$ statistic is greater than $t$ critical value. These variables were found to be minimum significant and so final modelling was done using these variables. Accepted gap and lag was considered as one independent variable in final modelling, since either accepted gap or lag will be present for each driver. The critical gap was modelling using independent variables accepted gap/lag and merging time. Modelling was done using 170 samples. The proposed model for critical gap estimation is shown below.

$\mathrm{T}_{\mathrm{c}}=1.217+0.306 \mathrm{~T}_{\mathrm{a}}+0.278 \mathrm{~T}_{\mathrm{mg}} \mathrm{R}^{2}=0.715$

Where $\mathrm{T}_{\mathrm{c}}=$ critical gap; $\mathrm{T}_{\mathrm{a}}=$ accepted gap; and $\mathrm{T}_{\mathrm{mg}}=$ merging time.

\section{Conclusion}

The U-turning behaviour of vehicles at selected median openings was studied. In the present study traditional, Modified Raff and merging behaviour approach were used for critical gap estimation The critical gap estimated by merging behaviour was found to be higher than that obtained by other methods. Critical gap estimated by merging behaviour approach is about $25.75 \%$ higher than traditional method and $28.09 \%$ higher than modified Raff method. The extremely lower critical gap values obtained by other methods show its inadequacy under heterogeneous traffic conditions. Merging behaviour was found to be more suitable under heterogeneous traffic conditions. Critical gap varies with vehicle category and critical gap of two wheeler is found to be minimum along all vehicle categories. As the size of vehicle increases the critical gap is also found to increase. A model was developed considering all modes, where critical gap of U-turning vehicles depends on accepted gap and merging time.

\section{References}

Ashalatha, R.; Chandra, S. 2011. Critical Gap through Clearing Behavior of Drivers at Unsignalised Intersections, KSCE Journal of Civil Engineering 15(8): 1427-1434.

Dash, S.; Mohapatra, S. S.; Dey, P. P. 2019. Estimation of Critical Gap of U-Turns at Uncontrolled Median Openings, Transportation Letters 11(5): 229-240.

Datta, S. 2014. Critical Gap Comparison between HARDERS and INAFOGA Methods for U-Turn Median Openings. In Proceedings of National Conference on Advances in Engineering and Technology, 1-9.

Datta, S.; Bhuyan, P. K. 2014. Which Method is Appropriate- INAFOGA or Probability Equilibrium for Critical Gap Estimation of U-turn Vehicles at Median Openings in Urban Indian Context? In Proceedings of the International Conference on Advances in Engineering and Technology, 459-465.

HCM. 2010. Highway Capacity Manual. TRB, National Research Council, Washington, D. C.

Jenjiwattanakul, T.; Sano, K. 2011. Effect of Waiting Time on the Gap Acceptance Behaviour of U-turning Vehicles at Midblock Median Openings. In Proceedings of the $9^{\text {th }}$ International Conference of Eastern Asia Society for Transportation Studies, 1601-1613. 
Khan, M. R.; Chalumuri, R. S.; Senapathi, V. 2015. Modelling of the Gap Phenomena at U-turn Provisions on the Median Openings of Inter-Urban Highway Corridors, Journal of the Eastern Asia Society for Transportation Studies 11: 1842-1856.

Liu, P.; Wang, X.; Lu, J.; Sokolow, G. 2007. Headway Acceptance Characteristics of U-Turning Vehicles at Unsignalized Intersections, Transportation Research Record 2027(1): 52-57.

Obaidat, T. I.; Elayan, M. S. 2013. Gap Acceptance Behavior at U-turn Median Openings - Case Study in Jordan, Jordan Journal of Civil Engineering 7(3): 332-341.

Sharma, V. K.; Mondal, S.; Gupta, A. 2017. Analysis of U-Turning Behaviour of Vehicles at Mid-Block Median Opening in Six Lane Urban Road: A Case Study, International Journal for Traffic and Transport Engineering 7(2): 243-255. 